\title{
Estudio acústico-estructural de la cabina de un vehículo automotriz
}

\section{Acoustic-Structural Study for the Cabin of an Automotive Vehicle}

\author{
L.A. Aguilera-Cortés \\ Universidad de Guanajuato, Campus Irapuato-Salamanca. \\ Salamanca, Gto. \\ E-mail:aguilera@salamanca.ugto.mx \\ A.L. Herrera-May \\ Universidad de Guanajuato, Campus Irapuato-Salamanca. \\ Salamanca, Gto. \\ Centro de Investigación en Micro y Nanotecnología de la Universidad Veracruzana, Boca del Río, Ver. \\ E-mail:leherrera@uv.mx \\ M. Torres-Cisneros \\ Universidad de Guanajuato, Campus Irapuato-Salamanca. \\ Salamanca, Gto. \\ E-mail:mtorres@salamanca.ugto.mx \\ M.A. González-Palacios \\ Universidad de Guanajuato, Campus Irapuato-Salamanca. \\ Salamanca, Gto. \\ E-mail:maxgopa@salamanca.ugto.mx \\ E.J. González-Galván \\ Centro de Investigación y Estudios de Posgrado, \\ Facultad de Ingeniería, Universidad Autónoma de San Luis Potosi, \\ San Luis Potosí, SLP, México. \\ E-mail:egonzale@uaslp.mx
}

(Recibido: enero de 2008; aceptado: enero de 2009)

\section{Resumen}

El estudio acústico-estructural acoplado de una cabina tipo automóvil mediante un nuevo modelo de elementos finitos en $3 \mathrm{D}$ es presentado. Este modelo, desarrollado con el software ANSYS, tiene una configuración geométrica sencilla que proporciona los modos de vibración acústico, estructural y acoplado de la cabina en un tiempo mínimo de cómputo. Además, el modelo obtiene fácilmente las respuestas del cambio en la presión acústica del fluido interior y los esfuerzos en la estructura de la cabina , provocados por una perturbación armónica. Los resultados de los primeros modos de vibración del sistema acoplado son del tipo rígido y con frecuencias menores a $5 \mathrm{~Hz}$. En cambio, los principales modos acústicos se presentan a frecuencias mayores de $87 \mathrm{~Hz}$, a excepción del modo Helmholtz $(0 \mathrm{~Hz})$. La máxima presión acústica en el rango de 2 a $400 \mathrm{~Hz}$ es de $108.70 \mathrm{~dB}$ para una excitación armónica de 1 N. La estructura de la cabina registra un esfuerzo máximo de $1.85 \mathrm{MPa}$ en la frecuencia de $51 \mathrm{~Hz}$. El modelo propuesto contribuye significativamente en la evaluación de zonas potencialmente críticas de ruido y esfuerzos en la cabina convencional de un vehículo automotriz.

Descriptores: cabina de vehículo automotriz, esfuerzo, método de elementos finitos, presiónacústica, sistemaacústico-estructural,vibraciones. 
DOI: http://dx.doi.org/10.22201/fi.25940732e.2010.11n1.007

Estudio acústico-estructural de la cabina de un vehículo automotriz

\begin{abstract}
An acoustic-structural study of a cabin for an automotive vehicle through a new $3 D$ finite element model is presented. This model, which is developed with software ANSYS, has a simple geometrical configuration to provide the uncoupled and coupled acoustic-structural modes of the cabin with computing minimum time. In addition, the model easily obtains the results of interior sound pressure and stress in the cabin caused by a harmonic excitation. The results of the first coupled-system modes are of rigid type and they have frequencies smaller than $5 \mathrm{~Hz}$. However, the main acoustic modes are present for frequencies greater than $87 \mathrm{~Hz}$, with the exception of Helmholtz mode $(0 \mathrm{~Hz})$. The highest acoustic pressure (in the range from 2 to 400 $\mathrm{Hz}$ ) is $107.8 \mathrm{~dB}$ for a harmonic excitation of $1 \mathrm{~N}$. The structure of the cabin registers a maximum stress of $1.85 \mathrm{MPa}$ to $51 \mathrm{~Hz}$. The model proposed significantly contributes in the evaluation of noise level and stress for an automotive vehicle.
\end{abstract}

Keywords: Cabin of an automotive vehicle, stress, finite element method, acoustic pressure, acoustic-structural system, vibrations.

\section{Introducción}

La estructura de una cabina de automóvil y el fluido interior (aire) constituye un sistema acústico-estructural acoplado. Varios métodos y técnicas han sido propuestos para analizar los sistemas acústicos estructurales acoplados y desacoplados, tales como métodos teóricos (Weiping et al., 2002, Luo et al., 1997, Scarpa, 2000), métodos de elementos finitos (Sandberg et al., 1998 y Sung et al., 1984) y modelos de elemento frontera (Goswami et al., 1990 y Yang et al., 1995).

En la actualidad, la disminución del ruido y vibración originados en la cabina de vehículos automotrices son parámetros muy importantes, debido a las regulaciones ambientales y al competitivo mercado mundial. Un buen diseño acústico de la cabina de un automóvil mejora su ambiente acústico y ocasiona una disminución en el nivel del ruido emitido hacia el exterior, lo cual mejora la calidad acústica ambiental. Debido a esto, los ingenieros de diseño y manufactura se enfocan en definir y optimizar las características de confort acústico y vibración de las cabinas de vehículos automotrices.

El ruido en la cabina de un automóvil puede ser resultado de excitaciones del motor, del sistema de transmisión, de la interacción llanta-carretera y el viento que se transmite hacia el interior del vehículo (Sung et al., 1984). Problemas de ruido no predecibles pueden ocurrir cuando las características vibratorias del sistema acústico-estructural no son diseñadas apropiadamente (Kim et al., 1998). El ruido generado por la vibración estructural de la cabina de vehículos (Nefske et al., 1982) es de especial interés a bajas frecuencias $(20-200 \mathrm{~Hz})$. Srinivasan et al. (2006) desarrollaron un modelo de orden reducido a baja frecuencia del análisis acústico estructural no amortiguado del interior de cavidades sostenidas por elementos flexibles. Hémon et al. (2004) estudiaron las oscilaciones de presión generadas por el flujo sobre cavidades en el contexto de vehículos automotrices.

La realización de pruebas experimentales para la medición del ruido en el interior de cabinas de automóviles generados por vibraciones, es muy costosa y requiere de procesos complicados y de alto consumo de tiempo. Una alternativa para predecir las vibraciones y el ruido en el interior de cabinas es mediante modelos de elementos finitos en 3D.

En este trabajo presentamos un estudio acústico-estructural de una cabina simplificada de un vehículo automotriz por medio de un nuevo modelo de elementos finitos en 3D. Este modelo, desarrollado con el software ANSYS, considera la interacción de la estructura de la cabina con el fluido interior que es ocasionada por excitaciones externas. El modelo propuesto predice fácilmente la variación de la presión acústica en el interior de la cabina, el cual es producido por perturbaciones armónicas con diferentes magnitudes y frecuencias.

\section{Formulación por elementos finitos}

Para una cavidad encerrada la ecuación de gobierno para la presión acústica $(P)$ sin disipación de energía en las fronteras (Condon et al., 1967) es:

$$
\{L\}^{T}(\{L\} P)-\frac{1}{c^{2}} \frac{\partial^{2} P}{\partial t^{2}}=0
$$

donde $c$ es la velocidad del sonido y el operador matricial está dado por 


$$
\{L\}^{T}=\left[\frac{\partial}{\partial x} \frac{\partial}{\partial y} \frac{\partial}{\partial z}\right]
$$

En la ecuación (1) la disipación viscosa ha sido despreciada, el fluido es considerado compresible, no hay flujo promedio del fluido, la densidad y presión a través de éste son uniformes.

Las condiciones de frontera para la presión acústica se determinan mediante consideraciones de la mecánica de fluidos. Para una superficie de frontera, $S$, que está en movimiento con una amplitud pequeña, un balance de momento requiere que

$$
\{n\} \cdot\{\nabla P\}=-\rho_{0}\{n\} \cdot \frac{\partial^{2}\{U\}}{\partial t^{2}},
$$

donde $n$ es la normal unitaria, $\rho_{0}$ es la densidad del fluido y $\{U\}$ es el vector de desplazamiento de la estructura.

Los elementos matriciales se encuentran al discretizar la ecuación de onda (1), utilizando el procedimiento de Galerkin (Zienkiewicz et al., 2000). Multiplicando la ecuación (1) por un cambio virtual en la presión $\{\delta P\}$ e integrando sobre el volumen $(V)$ de dominio, se obtiene

$$
\begin{aligned}
& \int_{V} \frac{1}{c^{2}} \delta P \frac{\partial^{2} P}{\partial t^{2}} d V+\int_{V}\left(\{L\}^{T} \delta P\right)(\{L\} P) d V= \\
& \left.\int_{S}\{n\}^{T} \delta P(\{L\}) P\right) d S
\end{aligned}
$$

donde $S$ es la superficie de interfase entre la estructura y el fluido.

Aplicando las condiciones de frontera de la ecuación (3) en la ecuación (4) se tiene

$$
\begin{aligned}
& \int_{V} \frac{1}{c^{2}} \delta P \frac{\partial^{2} P}{\partial t^{2}} d V+\int_{V}\left(\{L\}^{T} \delta P\right)(\{L\} P) d V= \\
& -\int_{S} \rho_{0} \delta P\{n\}^{T}\left(\frac{\partial^{2}}{\partial t^{2}}\{U\}\right) d S .
\end{aligned}
$$

La ecuación (5) contiene la presión del fluido $(P)$ y las componentes del desplazamiento mediante el vector $\{U\}$ que son las variables a encontrar. Las funciones de forma de los elementos finitos para la variación espacial de la presión y las componentes del desplazamiento están dadas por: $P=\{N\}^{T}\left\{P_{e}\right\}$ y $U=\left\{N^{\prime}\right\}^{T}\left\{U_{e}\right\}$. Si se aplica la segunda derivada con respecto al tiempo a las funciones de forma para sustituirlas en la ecuación (5) y si $[B]=\{L\}\{N\}^{T}$ y $\partial P=\{N\}^{T}\left\{\delta P_{c}\right\}$, entonces la ecuación (5) simplificada se puede expresar como

$$
\begin{aligned}
& \frac{1}{c^{2}} \int_{V}\{N\}\{N\}^{T} d V\left\{\ddot{P}_{e}\right\}+\int_{V}\{B\}^{T}[B] d V\left\{P_{e}\right\} \\
& +\rho_{0} \int_{S}\{N\}\{n\}^{T}\left\{N^{\prime}\right\}^{T} d S\left\{\ddot{U}_{e}\right\}=\{0\},
\end{aligned}
$$

donde $\left\{P_{e}\right\}$ es el vector de presión nodal, $\left\{U_{e}\right\}$ es el vector de desplazamiento nodal, $\{N\}$ es la función de forma de la presión y $\left\{N^{\prime}\right\}$ es la función de forma de los desplazamientos.

La ecuación (6) puede escribirse en la forma matricial convencional

$$
\left[M_{e}^{p}\right]\left\{\ddot{P}_{e}\right\}+\left[K_{e}^{p}\right]\left\{P_{e}\right\}+\rho_{0}\left[R_{e}\right]^{T}\left[\ddot{U}_{e}\right]=\{0\},
$$

donde $\left[M_{e}^{p}\right]$ es la matriz de masa del fluido, $\left[K_{e}^{p}\right]$ es la matriz de rigidez de la estructura y $\rho_{0}\left[R_{e}\right]^{T}$ es la matriz de masa acoplada (interfase estructura-fluido).

La ecuación matricial (7) no considera la disipación de energía, debida a la absorción de ésta en las fronteras. La energía disipada se obtiene al utilizar el término de disipación integrado sobre la superficie $(S)$ y utilizando la funciones de forma para la presión (ANSYS, 2002)

$$
D=\left\{\delta P_{e}\right\}^{T}\left(\frac{r}{\rho_{0} c}\right) \frac{1}{c} \int_{S}\{N\}\{N\}^{T} d S\left\{\dot{P}_{e}\right\}
$$

donde $r$ es la resistencia acústica del material de la estructura.

Este término, $D$, es adicionado a la ecuación matricial (7) para considerar la pérdida de energía en la superficie frontera (interfase estructura-fluido), como se muestra a continuación

$\left[M_{e}^{p}\right]\left\{\ddot{P}_{e}\right\}+\left[C_{e}^{p}\right]\left\{\dot{P}_{e}\right\}+\left[K_{e}^{p}\right]\left\{P_{e}\right\}+\rho_{0}\left[R_{e}\right]^{T}\left\{\ddot{U}_{e}\right\}=\{0\}$

donde $\left[C_{e}^{p}\right]$ es la matriz de amortiguamiento del fluido.

En el caso de interacción acústico-estructural, la carga de presión del fluido actuando en la interfase es adicionada a la ecuación matricial de elementos finitos de la estructura:

$$
\left[M_{e}\right]\left\{\ddot{U}_{e}\right\}+\left[C_{e}\right]\left\{\dot{U}_{e}\right\}+\left[K_{e}\right]\left\{U_{e}\right\}=\left\{F_{e}\right\}+\left\{F_{e}^{p r}\right\},(10)
$$


donde $\left[M_{e}\right]$ es la matriz de masa de la estructura, $\left[C_{e}\right]$ es la matriz de amortiguamiento de la estructura, $\left\{F_{e}\right\}$ es el vector de carga de excitación aplicada a la estructura $y\left\{F_{e}^{p r}\right\}$ es el vector que considera la carga de presión del fluido en la superficie de la interfase $(S)$ estructura- fluido. El vector de carga $\left\{F_{e}^{p r}\right\}$ es obtenido con el vector de presión nodal $\left(P_{\mathrm{e}}\right)$ y de la matriz de acoplamiento $\left[R_{\mathrm{e}}\right]$, la cual representa el área de la superficie efectiva asociada a cada nodo en la interfase estructura-fluido (ANSYS, 2002).

$$
\left\{F_{e}^{p r}\right\}=\left[R_{e}\right]\left\{P_{e}\right\}=\int_{S}\left\{N^{\prime}\right\}\{N\}^{T}\{n\} d S\left\{P_{e}\right\} .
$$

Sustituyendo la ecuación (11) en la (10) y considerando las cantidades de carga desconocidas en el miembro izquierdo de la ecuación (10), se obtiene la siguiente ecuación matricial de la estructura

$$
\left[M_{e}\right]\left\{\ddot{U}_{e}\right\}+\left[C_{e}\right]\left\{\dot{U}_{e}\right\}+\left[K_{e}\right]\left\{U_{e}\right\}-\left[R_{e}\right]\left\{P_{e}\right\}=\left\{F_{e}\right\} .
$$

Combinando las ecuaciones (9) y (12) se obtiene la ecuación matricial para el sistema acoplado estructura-fluido, dado de la siguiente forma

$$
\begin{aligned}
& {\left[\begin{array}{cc}
{\left[M_{e}\right]} & {[0]} \\
{\left[M^{f s}\right]} & {\left[M_{e}^{p}\right]}
\end{array}\right]\left\{\begin{array}{l}
\left\{\ddot{U}_{e}\right\} \\
\left\{\ddot{P}_{e}\right\}
\end{array}\right\}+\left[\begin{array}{cc}
{\left[C_{e}\right]} & {[0]} \\
{[0]} & \left\{C_{e}^{p}\right\}
\end{array}\right]\left\{\begin{array}{c}
\left\{\dot{U}_{e}\right\} \\
\left\{\dot{P}_{e}\right\}
\end{array}\right\}} \\
& +\left[\begin{array}{cc}
{\left[K_{e}\right]} & {\left[K^{f s}\right]} \\
{[0]} & {\left[K_{e}^{p}\right.}
\end{array}\right]\left\{\begin{array}{l}
\left\{U_{e}\right\} \\
\{P
\end{array}\right\}=\left\{\begin{array}{l}
\left\{F_{e}\right\} \\
\{0\}
\end{array}\right\},
\end{aligned}
$$

donde las expresiones del primer renglón de la ecuación matricial (13) representan al sistema estructural y las del segundo renglón refieren al sistema acústico. Con la finalidad de mantener una nomenclatura uniforme en las matrices de la ecuación (13) se utilizaron las siguientes relaciones

$$
\left[M^{f s}\right]=\rho_{0}\left[R_{e}\right]^{T} \text { y }\left[K^{f s}\right]=-\left[R_{e}\right],
$$

donde $\left[M^{\text {fs }}\right]$ es la matriz de masa acoplada y $\left[K^{f s}\right]$ es la matriz de rigidez acoplada.

La ecuación matricial (13) representa el acoplamiento de la estructura (cuerpo de la cabina) con el fluido (medio acústico encerrado). Éstas permiten obtener las frecuencias naturales, las configuraciones modales, así como la respuesta a una excitación del sistema acoplado. Para resolver estas ecuaciones pueden implementarse algoritmos que utilicen el método de elementos finitos o software comercial de elementos finitos. A continuación, se presentan los resultados de un estudio del acoplamiento acústico-estructural de la cabina de un vehículo automotriz, mediante un nuevo modelo de elementos finitos en 3D con el software ANSYS, el cual utiliza la formulación general de la interacción estructura-fluido que es representada por la ecuación matricial (13).

\section{Modelo cabina-fluido tipo automóvil}

El modelo de elementos finitos utilizado para estudiar la interacción estructura-fluido en la cabina de un automóvil considera un sistema de suspensión. El análisis modal del sistema estructural, acústico y acoplado es analizado en esta sección con el objetivo de encontrar sus configuraciones modales y frecuencias de resonancias en el rango de 0 a $400 \mathrm{~Hz}$. Además, se muestra la variación de la presión acústica y la distribución de los esfuerzos en la estructura de la cabina provocada por una excitación armónica.

\section{Características del modelo de elementos finitos}

La figura 1 muestra el modelo de la cabina de automóvil con dimensiones (figura 2) similares a las de un automóvil convencional con $1.36 \mathrm{~m}$ de ancho (Kim et al., 1998). La estructura de la cabina se contiene placas de acero de $4 \mathrm{~mm}$ de espesor con un módulo de elasticidad de $2.1 \times 10^{11} \mathrm{~Pa}$, una densidad de $7850 \mathrm{~kg} / \mathrm{m}^{3}$ y razón de Poisson de 0.3. Para el fluido interior se aplicaron las propiedades del aire con una densidad de $1.22 \mathrm{~kg} / \mathrm{m}^{3}$ y una velocidad de sonido de $343.50 \mathrm{~m} / \mathrm{s}$. En el sistema de suspensión se consideró una rigidez $k=18750 \mathrm{~N} / \mathrm{m}$ y un coeficiente de amortiguamiento $b=5324 \mathrm{Ns} / \mathrm{m}$ (dirección vertical), y en las otras dos direcciones $(\mathrm{x}, \mathrm{z}) \mathrm{de}$ $k=93750 \mathrm{~N} / \mathrm{m}$ y $b=2677 \mathrm{Ns} / \mathrm{m}$ (Dimarogonas, 1996). Además, se consideró un factor de pérdidas $\eta=0.001 \mathrm{pa-}$ ra las placas de acero (Beards, 1983).

Para obtener el coeficiente de absorción acústica $(\alpha)$ y el coeficiente de amortiguamiento estructural $(\beta)$ de las placas, se utilizaron las siguientes ecuaciones (Lyon y Dejong, 1995 y Dimarogonas, 1996):

$$
\alpha=\left(\frac{8 \pi f V}{c A}\right) \eta
$$


DOI: http://dx.doi.org/10.22201/fi.25940732e.2010.11n1.007

L.A. Aguilera-Cortés, A.L. Herrera-May, M. Torres-Cisneros, M.A. González-Palacios y E.J. González-Galván

$$
\beta=\frac{2 \zeta \omega-\alpha}{\omega^{2}},
$$

donde $V$ es el volumen de la cámara, $A$ es el área de todas las placas y $\zeta$ es la razón de amortiguamiento.

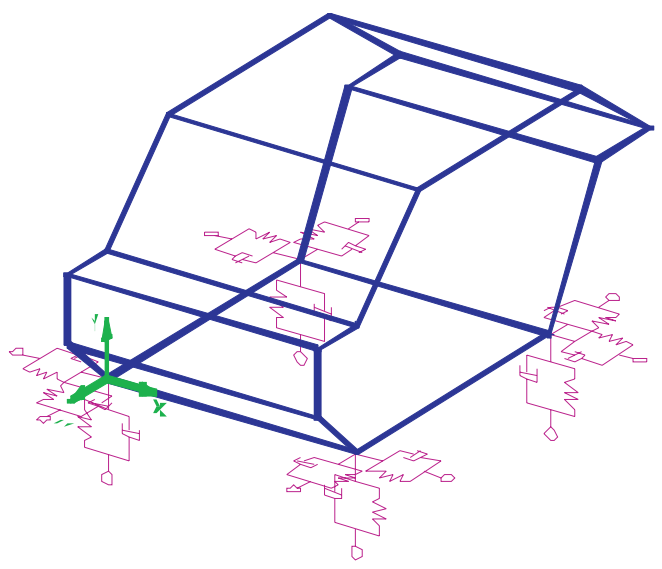

Figura 1. Esquema del modelo de una cabina de automóvil con sistema de suspensión

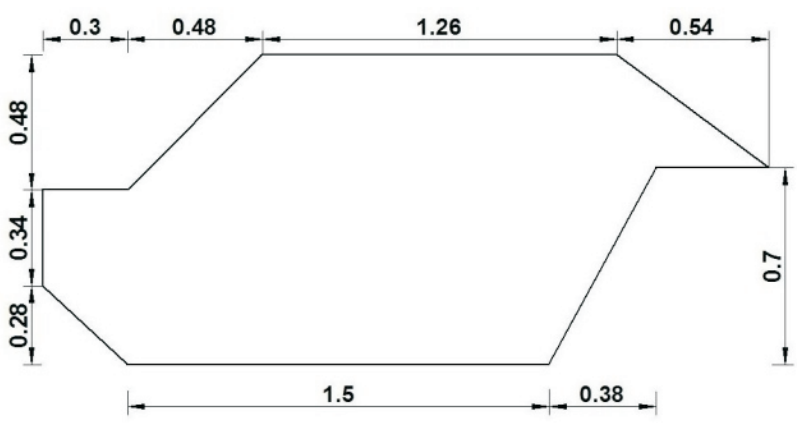

Figura 2. Dimensiones (m) del modelo de una cabina de automóvil (vista lateral)

El modelo de elementos finitos, desarrollado con el software ANSYS, utilizó los elementos shell63 para simular las placas de la estructura de la cabina. En cambio, para encontrar el comportamiento del fluido interior (aire) se consideraron los elementos fluid30 y en el sistema de suspensión (resorte-amortiguador) de la cabina se incluyeron elementos combin14. Para acoplar la interacción estructura-fluido se dispuso del acoplamiento acústico-estructural FSI de ANSYS.
Posteriormente, la estructura de la cabina acoplada con el fluido interior fue mallada con un total de 4614 nodos, como se muestra en la figura 3. Al final, se consideró una perturbación armónica con magnitud de $1 \mathrm{Ny}$ un rango de frecuencias de 2 a $400 \mathrm{~Hz}$. Esta perturbación fue aplicada próxima al centro de la placa inferior (nodo 467) de la cabina con la finalidad de conocer el comportamiento de la presión acústica en el interior de la cabina y la distribución de los esfuerzos en su estructura.

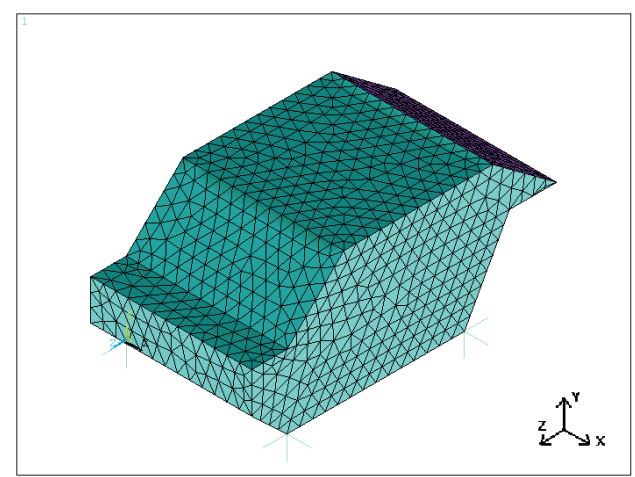

Figura 3. Modelo de elementos finitos de una cabina de automóvil

\section{Resultados}

En esta sección se presentan las respuestas del análisis modal del campo estructural, acústico y acoplado. Posteriormente, se muestran las variaciones de la presión acústica y los esfuerzos en la estructura de la cabina causados por una excitación armónica.

\section{Análisis modal de la cámara}

Primero se realizó el análisis modal de la estructura de la cabina y se obtuvieron las primeras 40 frecuencias de resonancia, como se muestran en la tabla 1. Las primeras seis configuraciones modales son del tipo rígido y el primer modo flexionante tiene una frecuencia de 13.16 $\mathrm{Hz}$. Las diferencias en las magnitudes de las frecuencias de resonancias son pequeñas, debido al delgado espesor de las placas (menor rigidez) y al sistema de suspensión.

Posteriormente, se efectuó el análisis modal del campo acústico de la cabina y se encontraron sus primeras 40 frecuencias de resonancia (tabla 2). La figura 4 muestra el primer modo del campo acústico a una frecuencia de $0 \mathrm{~Hz}$. Este modo es llamado Helmholtz (Ma et al., 1991) el cual es un modo de presión uniforme (modo de cuerpo rígido). Las siguientes seis configuraciones 
modales del campo acústico se visualizan en la figura 5 . En esta figura se observa que el segundo modo es del tipo longitudinal con las superficies nodales en formas casi verticales y orientadas perpendiculares a la dirección del eje z del compartimiento. En este modo la cara frontal y la cara superior derecha tienen una configuración modal más significativa en comparación con la región central del campo acústico de la cabina. Esto indica posibles regiones con alto nivel de ruido en los extremos de la cabina y bajo nivel de ruido en el centro de la cabina. Por lo tanto, para garantizar un confort acústico en la cabina del automóvil se debe evitar la presencia de perturbaciones con frecuencias cercanas a su resonancia. Si no es posible, entonces es conveniente adicionar elastómeros o realizar cambios en la estructura de la cabina para minimizar el ruido.

Los ingenieros de diseño y manufactura pueden auxiliarse del análisis modal propuesto en la etapa del diseño acústico de cabinas de automóviles para conocer las posibles regiones de ruido acústico.

Tabla 1. Primeras frecuencias de resonancia de la estructura del modelo de elementos finitos de una cabina de automóvil

\begin{tabular}{cccccccc}
\hline Núm. & $\mathrm{Hz}$ & Núm. & $\mathrm{Hz}$ & Núm. & $\mathrm{Hz}$ & $\mathrm{Núm.}$ & $\mathrm{Hz}$ \\
\hline 1 & 1.11 & 11 & 26.43 & 21 & 43.83 & 31 & 58.57 \\
2 & 1.21 & 12 & 27.59 & 22 & 45.81 & 32 & 60.18 \\
3 & 1.59 & 13 & 28.05 & 23 & 45.84 & 33 & 62.50 \\
4 & 3.96 & 14 & 32.47 & 24 & 47.00 & 34 & 62.56 \\
5 & 4.22 & 15 & 32.62 & 25 & 48.69 & 35 & 64.85 \\
6 & 4.70 & 16 & 32.87 & 26 & 50.82 & 36 & 68.32 \\
7 & 13.16 & 17 & 34.16 & 27 & 51.23 & 37 & 69.89 \\
8 & 16.26 & 18 & 40.34 & 28 & 55.15 & 38 & 71.12 \\
9 & 18.42 & 19 & 40.53 & 29 & 56.22 & 39 & 73.54 \\
10 & 19.18 & 20 & 42.36 & 30 & 57.64 & 40 & 74.73 \\
\hline
\end{tabular}

Tabla 2. Primeras frecuencias de resonancia del campo acústico del modelo de elementos finitos de una cabina de automóvil

\begin{tabular}{cccccccc}
\hline Núm. & $\mathrm{Hz}$ & Núm. & $\mathrm{Hz}$ & Núm. & $\mathrm{Hz}$ & $\mathrm{Núm}$. & $\mathrm{Hz}$ \\
\hline 1 & 0.00 & 11 & 247.93 & 21 & 324.78 & 31 & 388.08 \\
2 & 87.54 & 12 & 255.34 & 22 & 335.22 & 32 & 397.72 \\
3 & 126.65 & 13 & 267.65 & 23 & 336.58 & 33 & 398.33 \\
4 & 148.76 & 14 & 270.52 & 24 & 349.18 & 34 & 400.15 \\
5 & 154.28 & 15 & 273.69 & 25 & 350.73 & 35 & 408.64 \\
6 & 166.19 & 16 & 297.07 & 26 & 358.20 & 36 & 418.13 \\
7 & 196.04 & 17 & 297.25 & 27 & 361.65 & 37 & 418.42 \\
8 & 209.82 & 18 & 303.22 & 28 & 377.21 & 38 & 424.02 \\
9 & 211.81 & 19 & 307.20 & 29 & 379.39 & 39 & 426.39 \\
10 & 234.22 & 20 & 323.32 & 30 & 382.04 & 40 & 428.89 \\
\hline
\end{tabular}




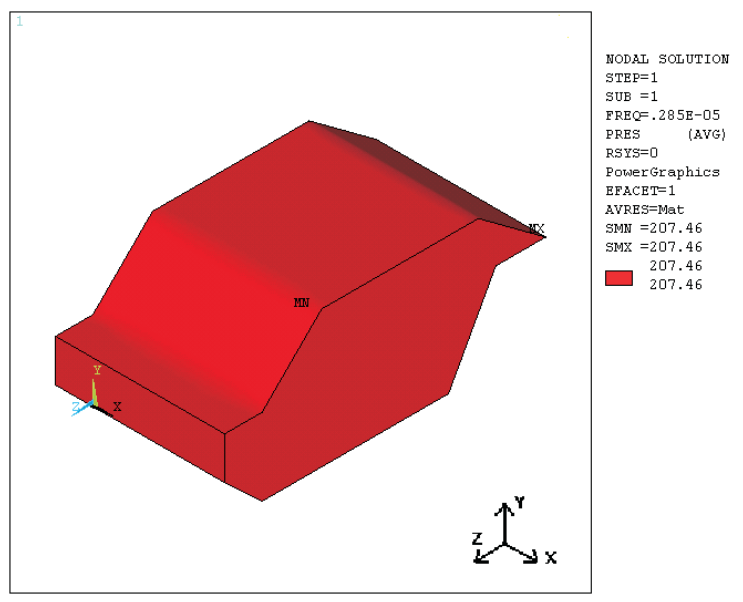

Figura 4. Modo Helmholtz del campo acústico del modelo de elementos finitos de una cabina de automóvil
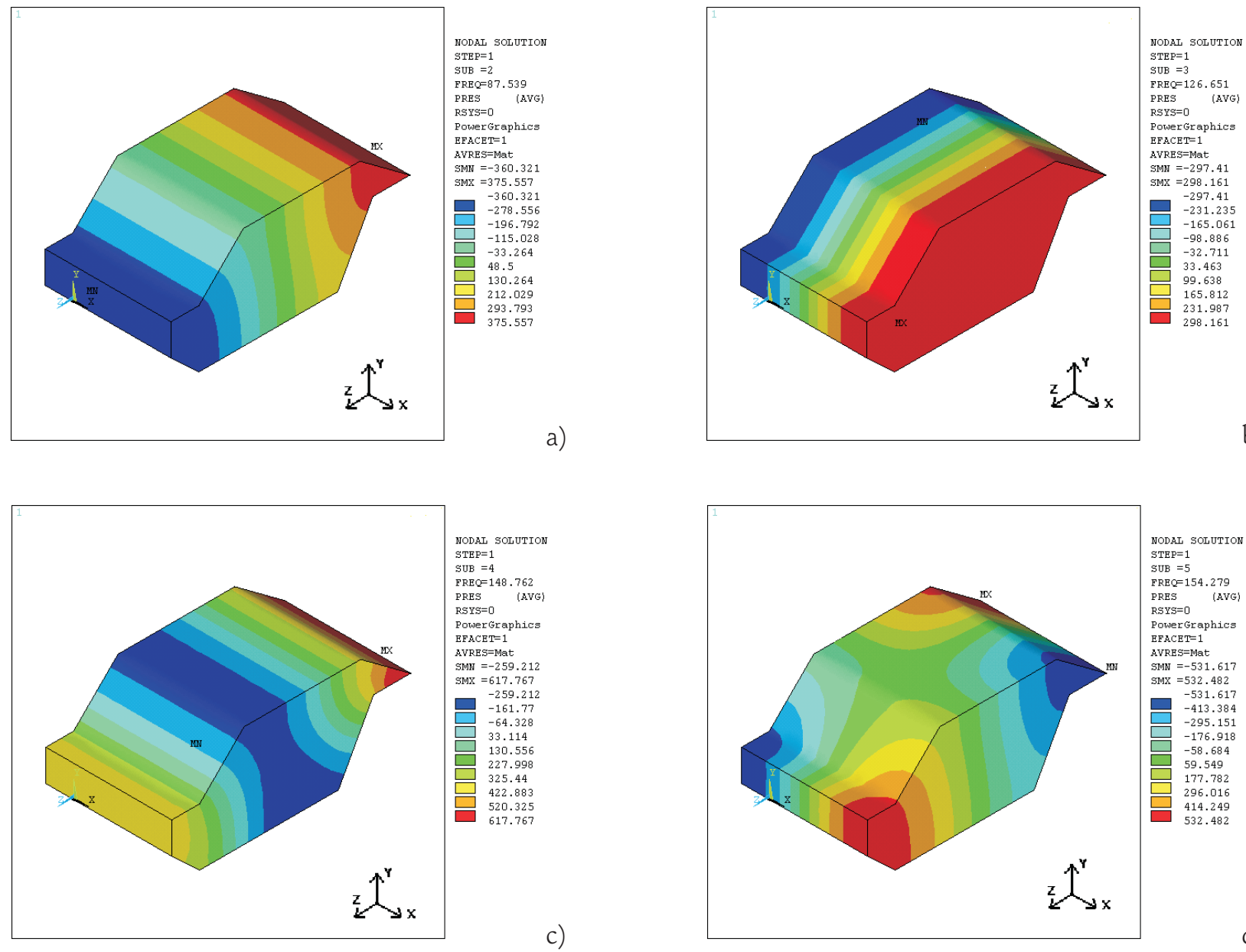

Figura 5. Ilustraciones del a) segundo, b) tercero, c) cuarto, d) quinto, e) sexto y f) séptimo modo acústico del modelo de elementos finitos de una cabina de automóvil (continua...) 

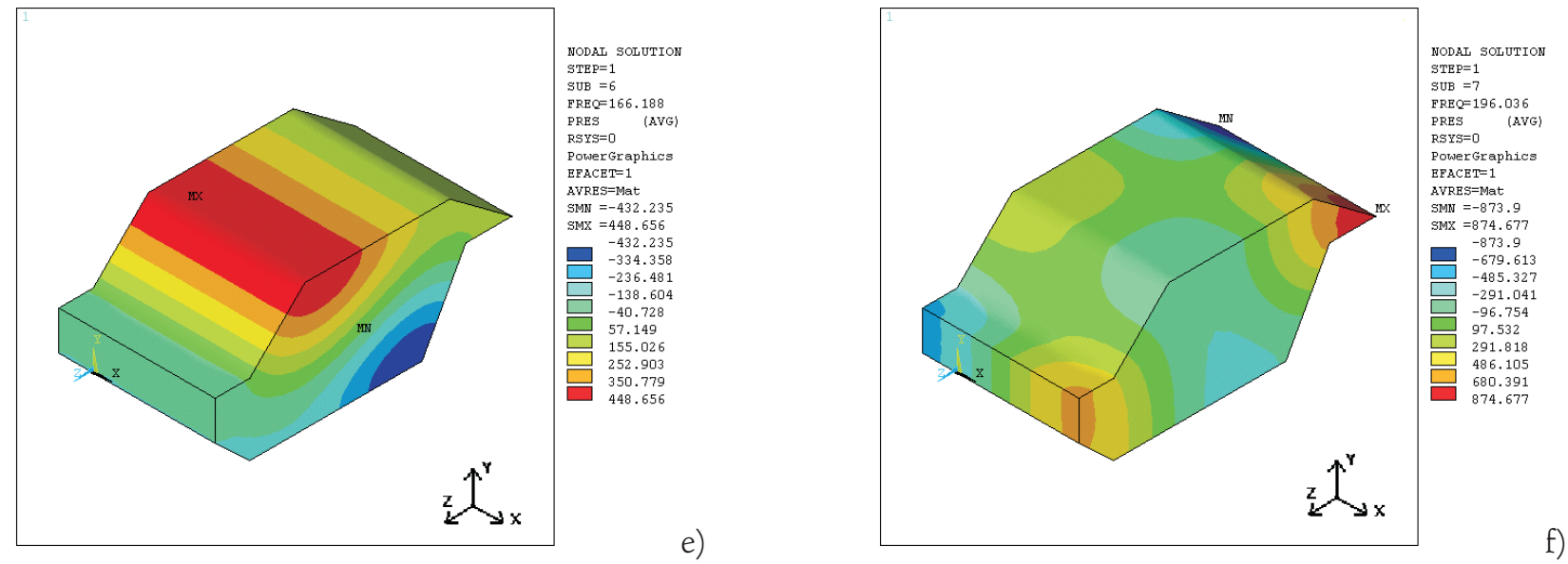

Figura 5. Ilustraciones del a) segundo, b) tercero, c) cuarto, d) quinto, e) sexto y f) séptimo modo acústico del modelo de elementos finitos de una cabina de automóvil (... continuación)

El siguiente análisis modal consideró el acoplamiento acústico-estructural de la cabina, obteniendo los primeros 15 modos de vibración del sistema acústico-estructural acoplado, como se indica en la tabla 3. La primera frecuencia natural ocurre a $0 \mathrm{~Hz}$ (figura 6), lo que indica un dominio del campo acústico. La figura 7 muestra las siguientes seis configuraciones modales en donde el segundo modo es rígido del tipo bamboleo y el quinto es rígido tipo rotación con respecto al eje vertical. La sexta configuración modal es del tipo bamboleo inverso. En cambio, el séptimo modo es rígido del tipo inclinación. Lo anterior concuerda con los resultados reportados por Tristan 2000, que distinguen a una primer zona (de 1 a $5 \mathrm{~Hz}$ ) relacionada con los modos rígidos.

En las primeras frecuencias de resonancia del sistema acoplado, el campo estructural tiene una mayor influencia, a excepción de la primera frecuencia en donde predomina el campo acústico.

Esto se origina porque las primeras frecuencias del campo estructural son de menor magnitud que la acústica (mayores de $87 \mathrm{~Hz}$ ).

Este análisis modal contribuye a visualizar posibles zonas de ruido en las cabinas de automóviles cuando existen perturbaciones armónicas en su estructura con frecuencias cercanas a su resonancia.

Tabla3. Primeras frecuencias de resonancia del sistema acústico-estructural acoplado del modelo de elementos finitos de una cabina de automóvil

\begin{tabular}{cccccc}
\hline Núm. & $\mathrm{Hz}$ & Núm. & $\mathrm{Hz}$ & Núm. & $\mathrm{Hz}$ \\
\hline 1 & 0 & 6 & 4.21 & 11 & 21.74 \\
2 & 1.11 & 7 & 4.69 & 12 & 26.54 \\
3 & 1.21 & 8 & 13.25 & 13 & 27.49 \\
4 & 1.58 & 9 & 16.20 & 14 & 28.03 \\
5 & 3.96 & 10 & 18.34 & 15 & 32.43 \\
\hline
\end{tabular}




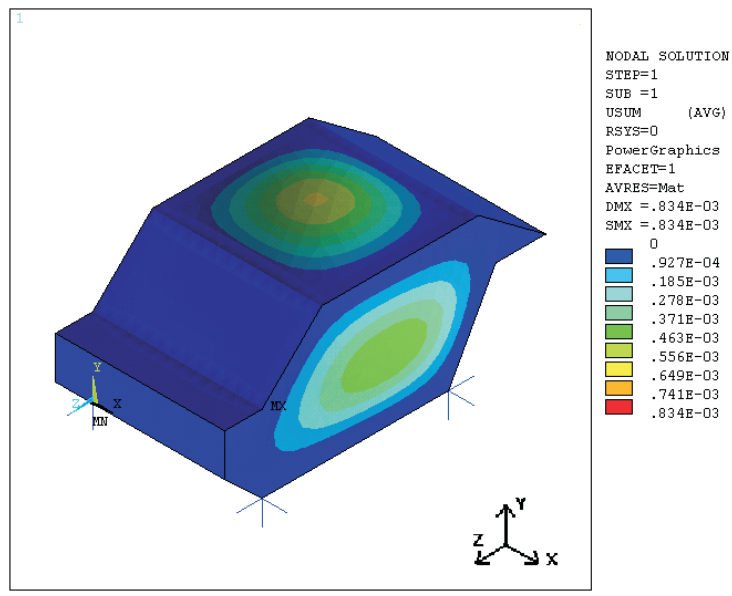

Figura 6. Primer modo de vibración del sistema acústico-estructural acoplado del modelo de elementos finitos de una cabina de automóvil.
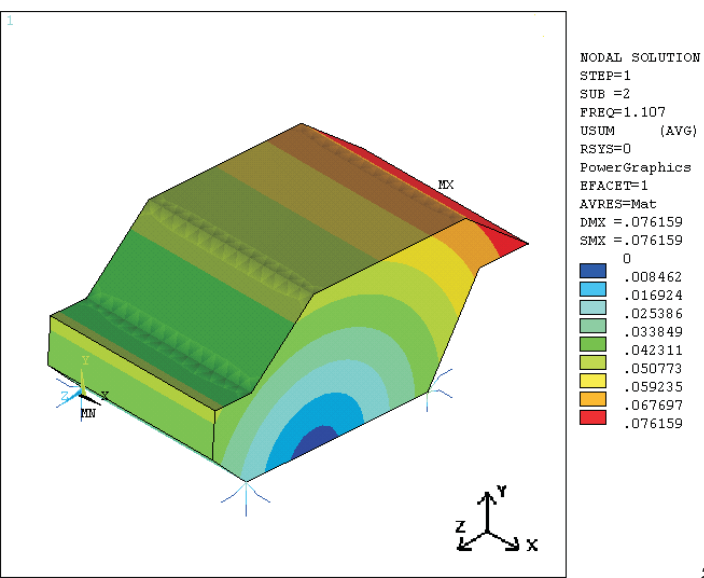

a)
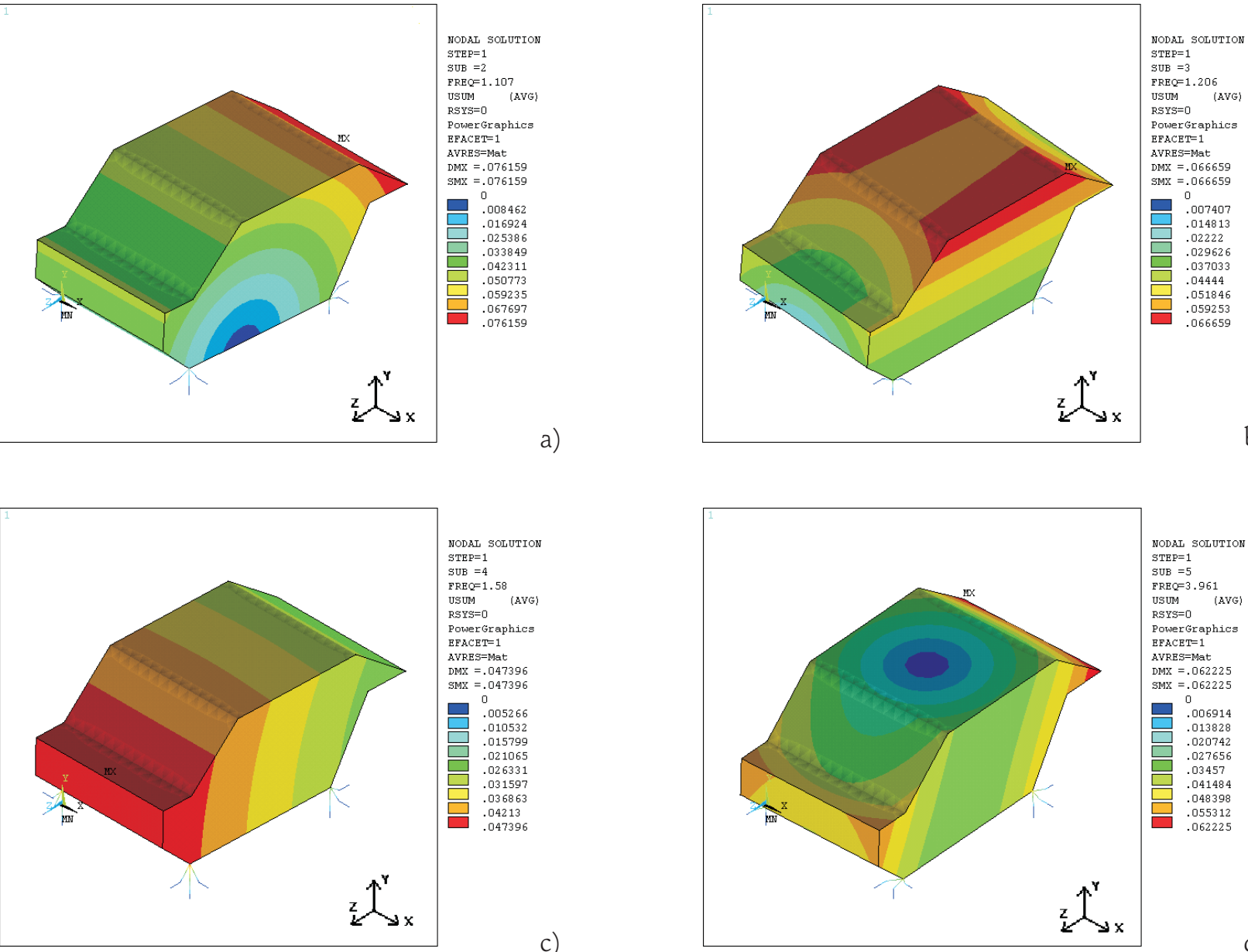

c)

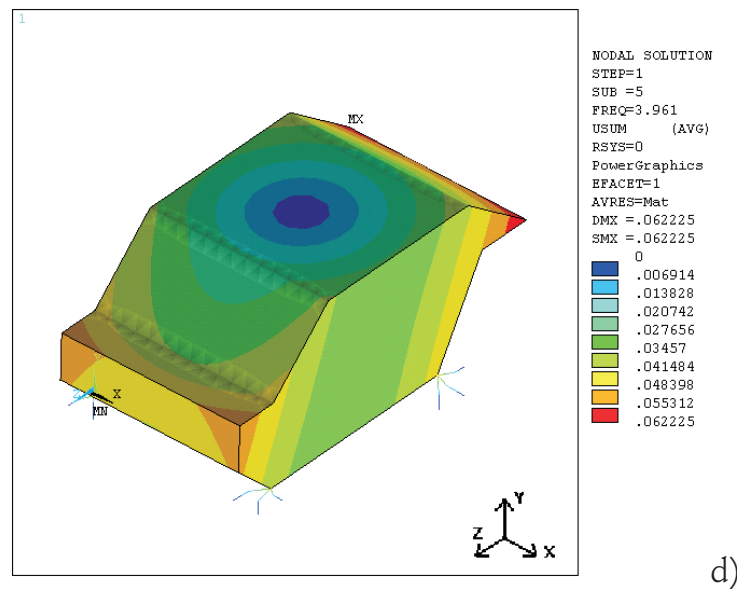

d)

Figura 7. Ilustraciones del a) segundo, b) tercero, c) cuarto, d) quinto, e) sexto y f) séptimo modo del sistema acústico-estructural acoplado del modelo de elementos finitos de una cabina tipo automóvil 

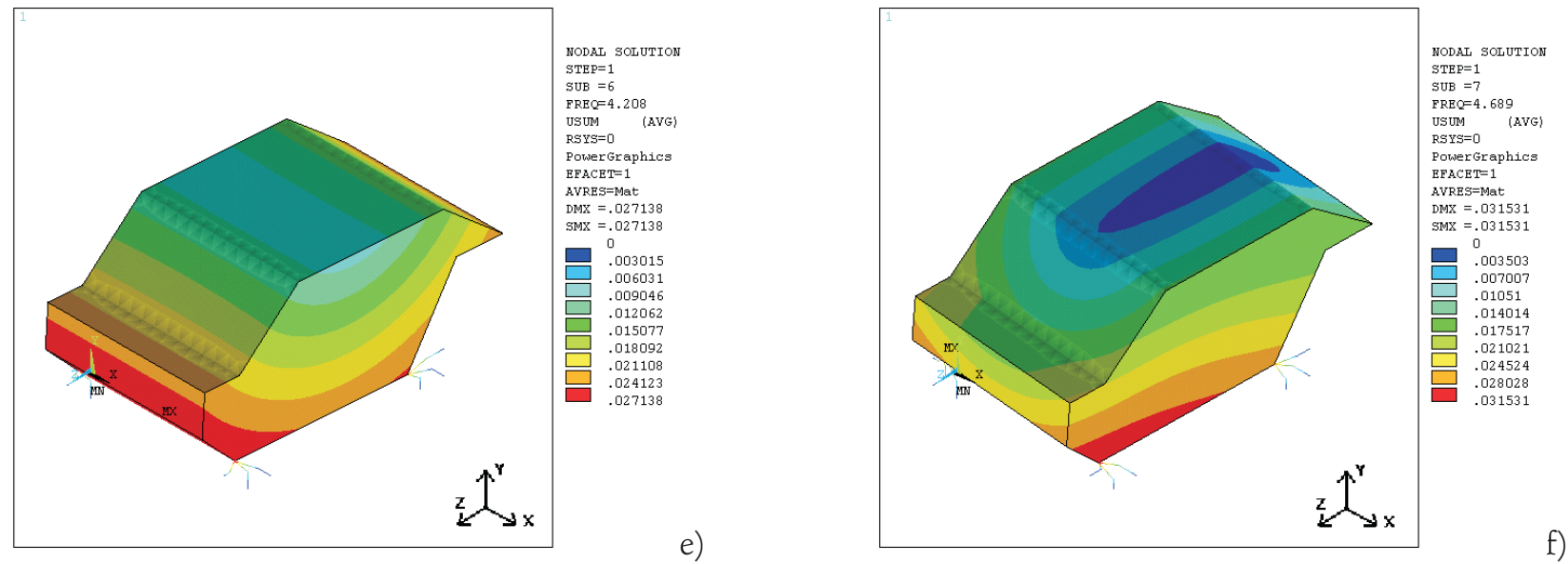

Figura 7. Ilustraciones del a) segundo, b) tercero, c) cuarto, d) quinto, e) sexto y f) séptimo modo del sistema acústico-estructural acoplado del modelo de elementos finitos de una cabina tipo automóvil (... continuación)

\section{Análisis armónico}

Una excitación armónica de $1 \mathrm{~N}$ fue aplicada en el nodo 647 que está ubicado cerca del centro geométrico de la placa inferior de la cabina. Dos casos fueron analizados, el primero consideró la excitación en un rango de frecuencias de 2 a $400 \mathrm{~Hz}$ y el segundo a una frecuencia específica de $196.77 \mathrm{~Hz}$. El objetivo de este análisis es encontrar los niveles de presión acústica y la distribución de los esfuerzos de Von Mises provocados por la excitación armónica.

En el primer caso se aplicó la excitación armónica puntual en la placa inferior de la cabina en un rango de 2 a $400 \mathrm{~Hz}$. Mediante las ecuaciones (15) y (16) se obtuvo el coeficiente de absorción acústica $\alpha=3.16 \times 10^{-3}$ (considerando una frecuencia promedio de $200 \mathrm{~Hz}$ ), un coeficiente de amortiguamiento estructural en las placas de $\beta_{\text {placa }}=7.94 \times 10^{-7} \mathrm{~s}^{-1} \mathrm{y}$ en los resortes de $\beta_{\text {res }}=7.96 \times 10^{-7}$ $\mathrm{s}^{-1}$. Los niveles de presión acústica (en el nodo superior donde se aplicó la excitación) generados por la excitación armónica son mostrados en la figura 8. Esta figura muestra algunas regiones de frecuencia con valores altos de presión acústica.

La primera gran presión acústica ( $97.85 \mathrm{~dB}$ ) se presenta a la frecuencia de $13 \mathrm{~Hz}$ y el máximo nivel de presión acústica (108.7 dB) se alcanza a la frecuencia de 33 $\mathrm{Hz}$. Magnitudes muy similares se presentan a 80, 160, 307 y $333 \mathrm{~Hz}$, aproximadamente. La excitación aplicada en la estructura con estas frecuencias produce niveles altos de presión acústica que genera ruido en el interior de la cabina. Estas regiones de frecuencia son críticas y deben evitarse. Si la perturbación armónica es aplicada en otras posiciones de la estructura de la cabina, se producirán diferentes distribuciones de la presión en el interior de la cabina y cuyas magnitudes dependerán de la cercanía de la frecuencia de excitación con las frecuencias de resonancia del sistema acústico-estructural acoplado.

La figura 9 muestra la variación de la presión acústica en función de la frecuencia en el nodo 387, el cual está ubicado a $20 \mathrm{~cm}$ de la placa superior y junto a la placa derecha. La posición de este nodo coincide con la zona del oído de un posible conductor. Para frecuencuas menores a $200 \mathrm{~Hz}$, esta gráfica contiene tres regiones (de 22-33 Hz, 90-94 Hz y 161-164 Hz) con niveles elevados de presión acústica. Este análisis ayuda a la evaluación del ruido dentro de la cabina y al desarrollo de alternativas para disminuirlo tales como modificaciones en el sistema de suspensión, la introducción de elementos que absorban gran cantidad de ruido (elastómero, plástico) y modificaciones en la geometría y materiales de la cabina.

La excitación armónica ocasiona una distribución de esfuerzos en la estructura de la cabina. La figura 10 muestra la magnitud de los esfuerzos en el punto de aplicación (nodo 647) de la excitación armónica (próxima al centro de la placa inferior). El valor máximo de los esfuerzos (1.85 $\mathrm{MPa}$ ) es obtenido a la frecuencia de 51 $\mathrm{Hz}$ y el segundo mayor esfuerzo (1.05 $\mathrm{MPa})$ a la frecuencia de $162 \mathrm{~Hz}$.

Por último, se realizaron los análisis de la distribución de la presión acústica en el interior de la cabina y el 


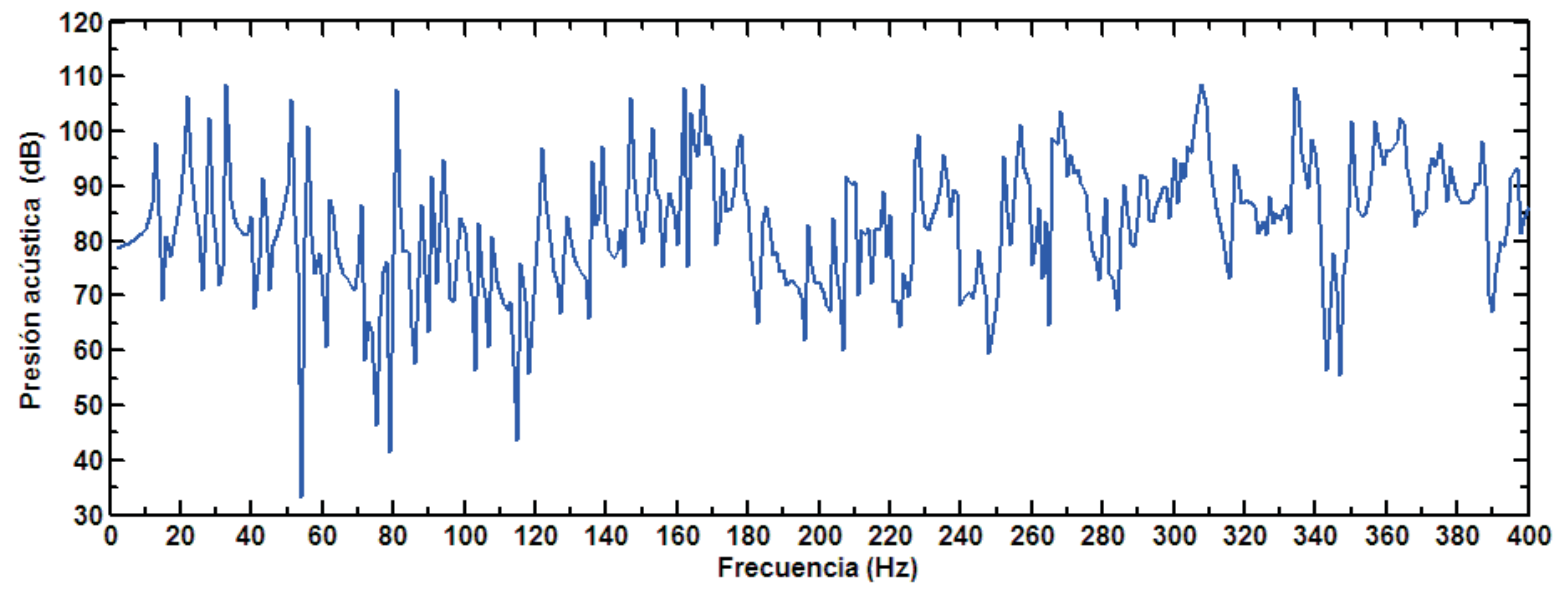

Figura 8. Respuesta de la presión acústica $(d B)$ del modelo de elementos finitos de una cabina de automóvil en el punto de aplicación de la excitación armónica de $1 \mathrm{~N}$

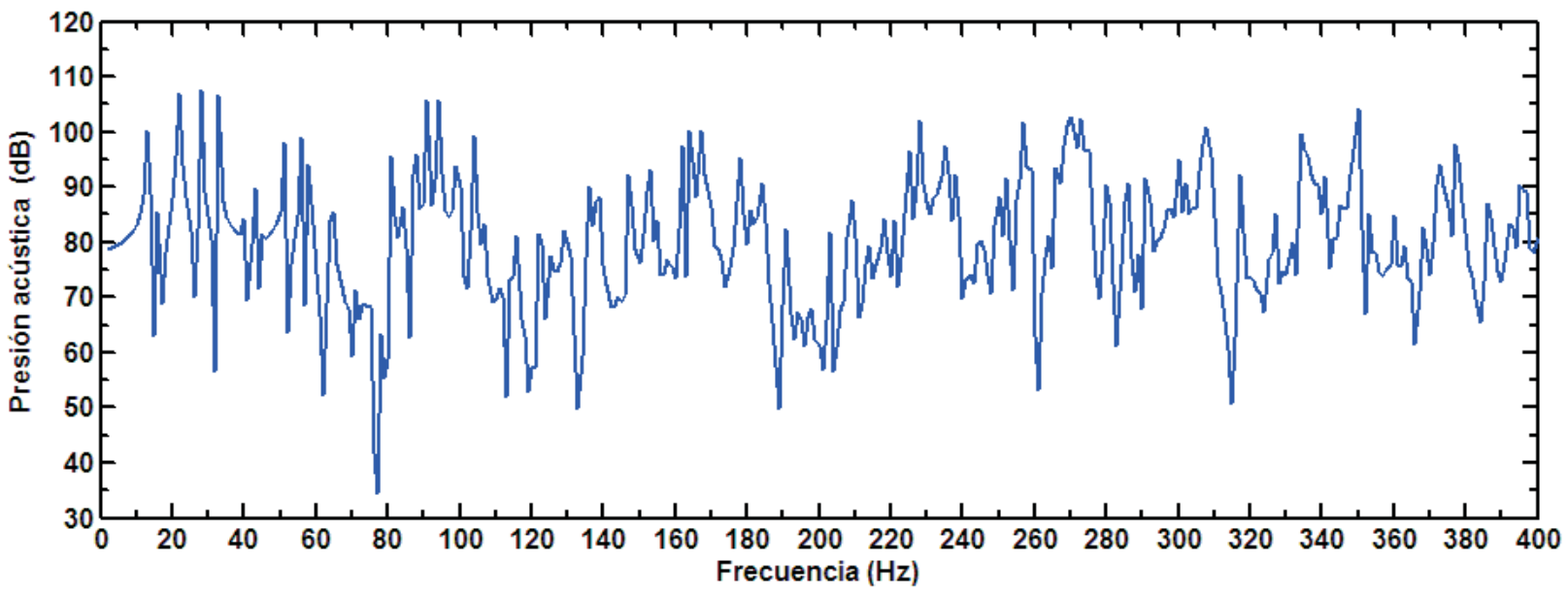

Figura 9. Respuesta de la presión acústica $(d B)$ del modelo de elementos finitos de una cabina de automóvil en el nodo 387 ubicado próximo al oído del conductor

comportamiento de los esfuerzos en su estructura a la frecuencia de $196.77 \mathrm{~Hz}$ (frecuencia de resonancia del sistema acústico-estructural acoplado). La amplitud de la excitación y su punto de aplicación (nodo 647) es la misma del caso anterior. A esta frecuencia se tiene que $\alpha=3.11 \times 10^{-3}, \beta_{\text {placa }}=8.07 \times 10^{-7} \mathrm{~s}^{-1}$ y $\beta_{\text {res }}=8.09 \times 10^{-7} \mathrm{~s}^{-1}$. La figura 11 muestra la distribución de la presión acústica en el interior de la cabina. El máximo nivel de presión de $86.89 \mathrm{~dB}$ se presenta cerca de la placa inferior, en algunas regiones próximas a la placa superior y en las esquinas de la cara posterior.

En este caso, el ruido en el punto de aplicación de la excitación es de 86.89 dB y en la posición del oído de un posible conductor (nodo 387) es de $63 \mathrm{~dB}$.
En comparación con las figuras 8 y 9 , se presenta una diferencia de $3.89 \mathrm{~dB}$ en el punto de aplicación de la excitación y de $2 \mathrm{~dB}$ en la posición del nodo 387 a la frecuencia de 196.77 Hz. En el segundo análisis armónico se visualiza la presión acústica en el interior de la cabina, pero a una sola frecuencia $(196.77 \mathrm{~Hz})$. En cambio, en el primer análisis armónico, se muestra la presión acústica en un rango amplio de frecuencias, aunque sólo en puntos específicos que el diseñador selecciona en la etapa de postproceso. La figura 12 muestra la distribución de los esfuerzos de Von Mises en la estructura de la cabina que son generados por la excitación armónica de $1 \mathrm{~N}$. El máximo esfuerzo es de $52.40 \mathrm{kPa}$ en el punto de aplicación de la excitación (nodo 647). 
DOI: http://dx.doi.org/10.22201/fi.25940732e.2010.11n1.007

Estudio acústico-estructural de la cabina de un vehículo automotriz

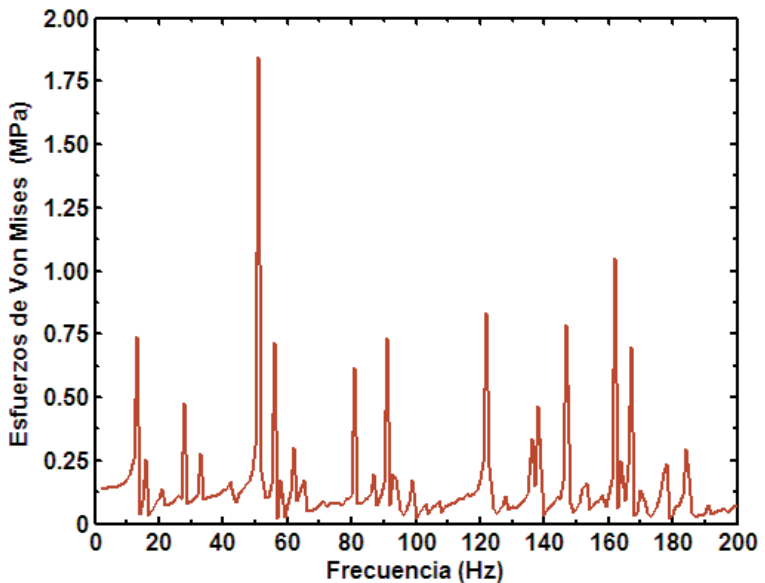

Figura 10. Respuesta de los esfuerzos de Von Mises (Pa) en el modelo de elementos finitos de una cabina de automóvil en el punto de aplicación de la excitación armónica de $1 \mathrm{~N}$
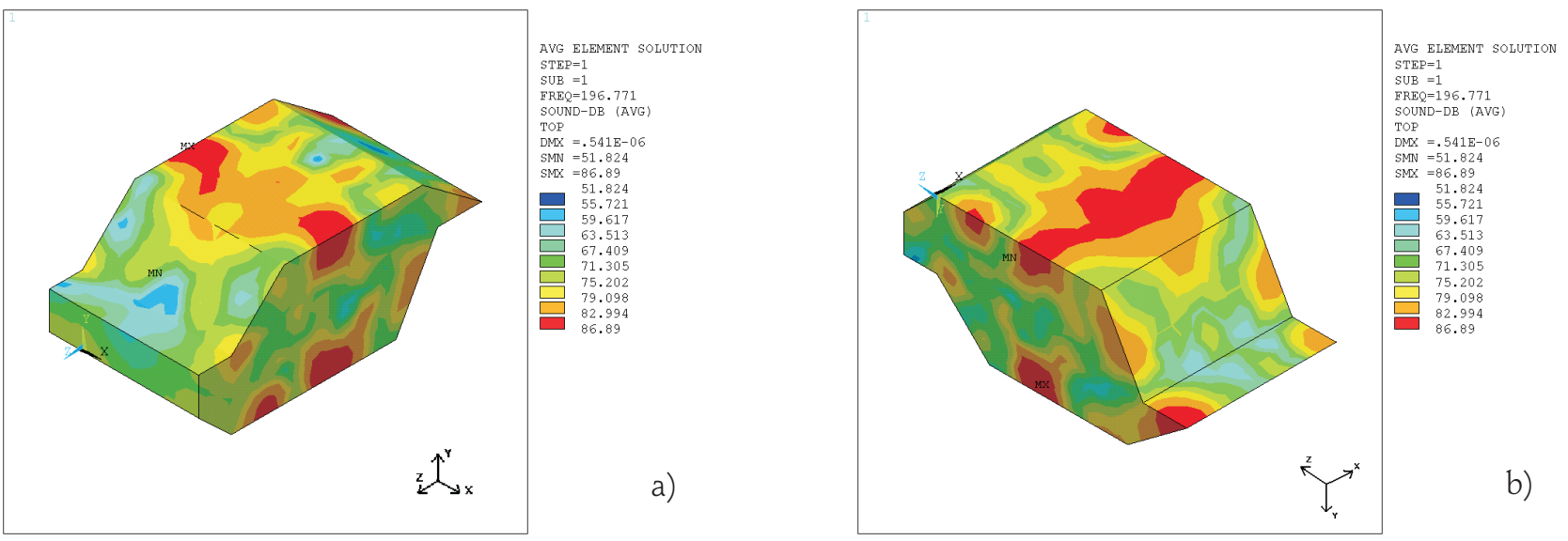

Figura 11. a) Vista superior e b) inferior de la distribución de la presión acústica (dB) en el interior de una cabina de automóvil provocado por una excitación armónica de $1 \mathrm{~N}$ a $196.77 \mathrm{~Hz}$
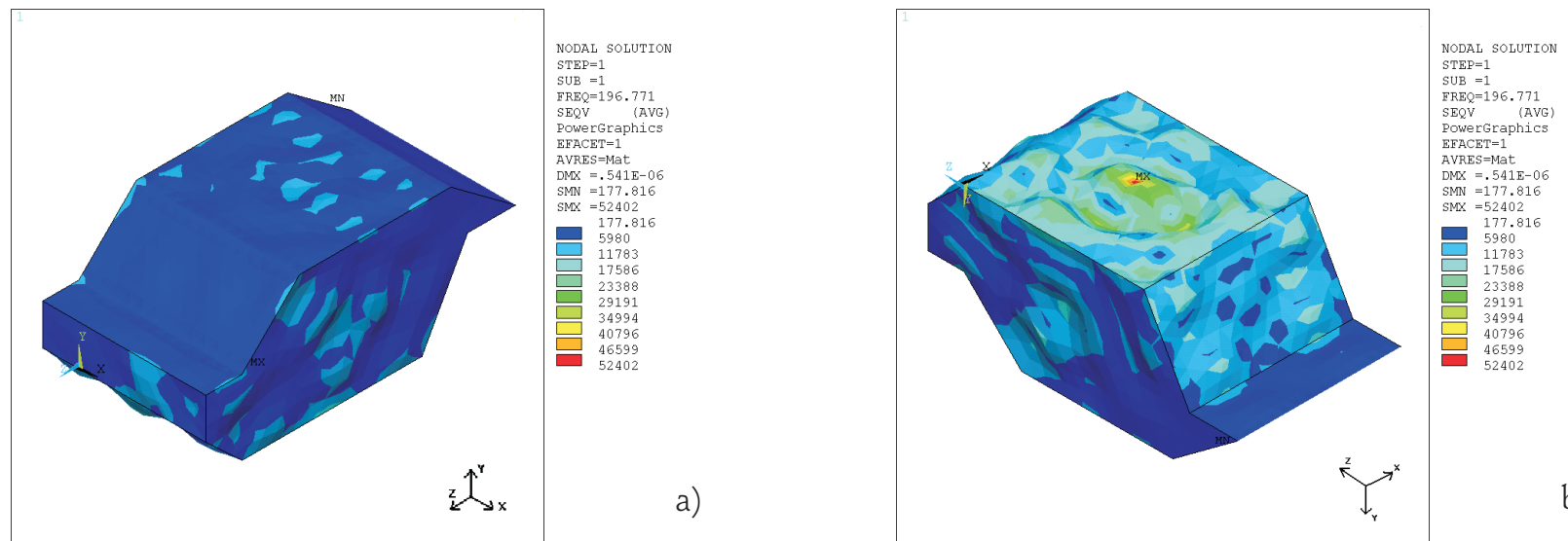

Figura 12. a) Vista superior e b) inferior de la distribución de los esfuerzos de Von Mises (Pa) en la estructura de la cabina de automóvil provocado por una excitación armónica de $1 \mathrm{~N}$ a $196.77 \mathrm{~Hz}$. 
DOI: http://dx.doi.org/10.22201/fi.25940732e.2010.11n1.007

L.A. Aguilera-Cortés, A.L. Herrera-May, M. Torres-Cisneros, M.A. González-Palacios y E.J. González-Galván

La placa inferior de la cabina es la que presenta las mayores magnitudes de los esfuerzos de Von Mises. Las placas superior y frontal tienen en general esfuerzos menores de $12 \mathrm{kPa}$.

\section{Conclusiones}

Un nuevo modelo de elementos finitos del acoplamiento acústico-estructural en $3 \mathrm{D}$ de una cabina convencional de automóvil fue diseñado para predecir el nivel de ruido y la distribución de los esfuerzos en la cabina. El modelo fue realizado con el software ANSYS y presentó una configuración geométrica simple. Mediante este modelo se obtuvieron los modos de vibración acústico, estructural y acoplado de la cabina del automóvil. La distribución de la presión acústica en el interior de la cabina, provocada por una perturbación armónica de $1 \mathrm{~N}$, fue analizada en un rango de frecuencias de 2 a $400 \mathrm{~Hz}$ y a una frecuencia específica $(196.77 \mathrm{~Hz})$ cercana a su frecuencia de resonancia acústica. La máxima presión acústica (108.70 dB) se registró en el punto de aplicación (cerca del centro geométrico de la placa inferior de la cabina) de la excitación armónica a una frecuencia de $33 \mathrm{~Hz}$. Además, en este punto fue obtenido el máximo esfuerzo de Von Mises (1.85 MPa) en la estructura de la cabina. El modelo propuesto puede ser utilizado fácilmente por diseñadores del sector automotriz para conocer las zonas críticas de ruido en el interior de una cabina convencional de automóvil, las cuales son producidas por diferentes excitaciones en la estructura de la cabina.

\section{Agradecimientos}

El segundo autor agradece al CONACYT por la beca otorgada durante la realización del trabajo. Este trabajo recibió apoyo parcial del CONACYT No. Convenio 53139.

\section{Referencias}

ANSYS, Manual de ANSYS5.7. 2002.

Beards C.F. Structural Vibration Analysis: Modelling, Analysis and Damping of Vibrating Structures. John New York, USA. Wiley \& Sons Inc.1983.

Condon E.U., Odishaw H. Handbook of Physics. 2nd Edition. New York, USA. McGraw-Hill. 1967.

Dimarogonas A.D. Vibrations for Engineers. 2nd Edition. New Jersey. USA. Prentice Hall. 1996.

Goswami P.P, Rudolphi T.J., Rizzo F.J., Shippy D.J. A Boundary Element Model for Acoustic-Elastic Interaction with
Applications in Ultrasonic NDE. Journal of Nondestructive Evaluation, 9:101-112. 1990.

Hémon P., Santi F., Amandolèse X. On the Pressure Oscillations Inside a Deep Cavity Excited by a Grazing Airflow. European Journal of Mechanics B/fluids, 23:617-632. 2004.

Kim S.H., Lee J.M. A Practical Method for Noise Reduction in a Vehicle Passenger Compartment. Journal of Vibration and Acoustics, 120:199-205. 1998.

Luo J., Gea H.C. Modal Sensitivity Analysis of Coupled Acoustic-Structural Systems. Journal of Vibration and Acoustics, 119:545-550. 1997.

Lyon R.H., Dejong R.G. Theory and Application of Statistical Energy Analysis. 2nd Edition. Boston, MA. USA. Butterworth-Heinemann. 1995.

Ma D.Z., Hagiwara I. Sensitivity Analysis Methods for Coupled Acoustic-Structural Systems Part I: Modal Sensitivities. AIAA Journal, 19:1787-1795. 1991.

Nefske D.J., Wolf J.A. Jr, Howell L.J. Structural-Acoustic Finite Element Analysis of the Automobile Passenger Compartment: a Review of Current Practice. Journal of Sound and Vibration, 80:247-266. 1982.

Sandberg J., Goransson P. A Symmetric Finite Element Formulation for Acoustic Fluid-Structure Interaction Analysis. Journal of Sound and Vibration,123:507-515.1998.

Scarpa F. Parametric Sensitivity Analysis of Coupled Acoustic-Structural Systems. Journal of Vibration and Acoustics, 122:109-115. 2000.

Srinivasan R, Monrrey D., Bell A., Durodola J.F., Rudnyi E.B., Korbink J.G. Compact Structural-Acoustic Coupled Models via Model Order Reduction (MOR). 13nd International Congress on Sound and Vibration, Vienna, Austria, julio 2-6, 2006.

Sung S.H., Nefske D.J.A. Coupled Structural-Acoustic Finite Element Model for Vehicle Interior Noise Analysis. Journal of Vibration, Acoustic, Stress, and Reliability in Design, 106:314-318. 1984.

Tristán A. Validación teórico-experimental para respuesta estructural modal en vehículos. Proceedings VI International Meeting of Mechanical Engineering, San Luis Potosí, México, pp. 121-130. 2000.

Weiping D., Hualing C. Research on the Interior Noise Contributed from a Local Panel's Vibration of an Elastic Thin-Walled Cavity. Applied Acoustics, 63:95-102. 2002.

Yang T.C., Tseng C.H., Ling S.F. A Boundary-Element-Based Optimization Technique for Design of Enclosure Acoustical Treatments. Journal of the Acoustical Society of America, 98:302-312. 1995.

Zienkiewicz O.C., Taylor R.L. The Finite Element Method, Volume 3: Fluid Dynamics.5th Edition, Oxford, Butterworth-Heinemann. 2000. 
DOI: http://dx.doi.org/10.22201/fi.25940732e.2010.11n1.007

Estudio acústico-estructural de la cabina de un vehículo automotriz

\section{Semblanza de los autores}

Luz Antonio Aguilera-Cortés. Obtuvo la licenciatura, maestría y doctorado en ingeniería mecánica en la Universidad de Guanajuato en 1988, 1990 y 1995, respectivamente. Ha realizado investigaciones en el análisis y síntesis de sistemas mecánicos y mecatrónicos, vibraciones mecánicas y MEMS. Tiene publicados 8 artículos en revistas internacionales, 10 en revistas nacionales y 60 artículos en foros con arbitraje a nivel nacional e internacional. Profesor con perfil PROMEP desde 1999. De 1992 a 1996, candidato a investigador nacional y actualmente SNI I. Actualmente adscrito a la FIMEE de la Universidad de Guanajuato con nombramiento de Profesor titular A.

Agustín Leobardo Herrera-May. Obtuvo la maestría en ingeniería mecánica en la Universidad de Guanajuato en 2002 y la licenciatura en ingeniería mecánica eléctrica en 2000 por la Universidad Veracruzana. Ha realizado investigaciones en el estudio de modelación, simulación y análisis con enfoque hacia el diseño mecánico, vibraciones mecánicas y elemento finito. Tiene el reconocimiento del Perfil deseable de PROMEP. Ha realizado 18 artículos para congresos nacionales e internacionales y 15 artículos de investigación y divulgación en revistas indizadas. Actualmente es estudiante de doctorado en ingeniería mecánica en la FIMEE de la Universidad de Guanajuato.

Miguel Torres-Cisneros. Se graduó como ingeniero en comunicaciones y electrónica en la Universidad de Guanajuato en 1987, de maestro en ciencias en CIO en 1991 y se doctoró en el INAOE en 1997. En 2002, realizó una estancia posdoctoral en la Universidad de Dayton. Desde hace 12 años es investigador titular en la Universidad de Guanajuato. Ha publicado 29 trabajos en revistas científicas con arbitraje. Ha publicado 46 artículos en extenso en congresos y 5 artículos de divulgación en revistas nacionales. Es investigador nacional (SNI) desde 1992, actualmente nivel II y es profesor con perfil PROMEP desde 1999.

Max Antonio Gonzalez-Palacios. Graduado en la Universidad Iberoamericana-León como licenciado en ingeniería mecánica y eléctrica en 1986. En 1989, obtuvo el grado de maestría en la FIMEE de la Universidad de Guanajuato, y en 1992 el de doctor por la Universidad McGill, Montreal, en donde completó un posdoctorado en 1993. De 1997 a 2000 , dirigió el Departamento de Investigación y Desarrollo de la Placage Unique, Inc., en Québec. Desde el 2000, es director de integración de procesos industriales, donde ha desarrollado diversos proyectos de investigación aplicada. Actualmente es profesor asociado de FIMEE de la Universidad de Guanajuato y pertenece al SNI (nivel I).

Emilio Jorge González-Galván. Recibió el grado de licenciatura y maestría en ingeniería mecánica en la FIMEE de la Universidad de Guanajuato en 1990 y 1991, respectivamente. Desde 1991 hasta 1996, realizó una estancia en la Universidad de Notre Dame, obteniendo el grado de doctor en ingeniería mecánica en 1995 y realizando un posdoctorado en la misma institución hasta 1996. En ese mismo año, se incorporó a la Facultad de Ingeniería de la Universidad Autónoma de San Luis Potosí, con cargo de profesor-investigador. Es autor de más de 60 trabajos científicos, es co-inventor en una patente asociada al control de robots y pertenece al SNI (nivel I). 